\title{
Mathematical Analysis of a Reactive Viscous Flow through a Channel Filled with a Porous Medium
}

\author{
Samuel O. Adesanya, ${ }^{1}$ J. A. Falade, ${ }^{2}$ J. C. Ukaegbu, ${ }^{1}$ and K. S. Adekeye \\ ${ }^{1}$ Department of Mathematical Sciences, Redeemer's University, Ede, Nigeria \\ ${ }^{2}$ Department of Physical Sciences, Redeemer's University, Ede, Nigeria \\ Correspondence should be addressed to Samuel O. Adesanya; adesanyaolumide@yahoo.com
}

Received 19 July 2016; Accepted 14 November 2016

Academic Editor: Ghulam Shabbir

Copyright ( 2016 Samuel O. Adesanya et al. This is an open access article distributed under the Creative Commons Attribution License, which permits unrestricted use, distribution, and reproduction in any medium, provided the original work is properly cited.

An investigation has been carried out to study entropy generation in a viscous, incompressible, and reactive fluid flowing steadily through a channel with porous materials. Approximate solutions for both velocity and temperature fields are obtained by using a rapidly convergent Adomian decomposition method (ADM). These solutions are then used to determine the heat irreversibility and Bejan number of the problem. Variations of other important fluid parameters are conducted, presented graphically, and discussed.

\section{Introduction}

Studies on heat irreversibility in moving fluid find its relevance in several geological, petrochemical, and industrial applications. In most flows at extremely high temperature, heat irreversibility is unavoidable. This usually leads to material waste due to reduced efficiency of the thermofluid machine. To conserve energy, Bejan [1] introduced an approach that is based on the thermodynamics second law to predict the performance of thermal systems so as to maximize scarce available energy for work and minimize wastages. Following his analysis, Al-Zaharnah and Yilbas [2] considered the irreversibility analysis in a viscous pipe flow. Haddad et al. [3] examined the heat irreversibility in forced convective flow in concentric cylindrical annulus under diverse flow conditions. Kahraman and Yürüsoy [4] applied the same approach to study the heat irreversibility in nonNewtonian fluid flow through pipes. Aksoy [5] considered the influence of couple stresses on the development of heat irreversibility in a channel with adiabatic surface and constant heat flux. Ting et al. [6] considered the irreversibility associated with nanofluids in a microchannel with porous materials using water-alumina. Moreover, Khan and Gorla [7] addressed the convective problem in non-Newtonian fluid flow through a channel with porous medium and heat flux. Revellin et al. [8] addressed the thermal performance of adiabatic two-phase flow using two different methods. Hedayati et al. [9] utilized the thermodynamics analysis to optimize flow on a nonstationary wedge. Butt and Ali [10] reported the irreversibility analysis of fluid slippage with convective boundaries. Other works that focused on the minimization of energy losses in a fluid flow can be found in references [11-19] and many more too numerous to be listed.

From applications' point of view, studies on transport reactive fluids in porous media are very important since they occur in many important areas like water treatment using fixed beds, agriculture, oil recovery, ground water flows, geothermal engineering, exhaust systems in combustion, material processing, and reservoir engineering. Recently, Rundora and his associates [20-22] documented several investigations on unsteady reactive fluid flow in porous medium and how the flow evolved to the steady state. Bég et al. [23] examined the flow of viscoelastic fluid through a nonDarcian porous medium. Makinde [24] studied the inherent heat irreversibility in reactive fluid through a channel filled with porous material.

In all the studies above, the entropy productions in the flow of viscous incompressible fluid flow through porous medium have not been investigated. Therefore, the work done 
in [24] can be further extended to give more interesting results on the thermodynamics and heat transfer properties of the fluid flow. This is because huge amount of money and effort could be wasted if the inherent irreversibility in the fluid flow is not well addressed. Therefore, the specific objective of this article is to examine the rate at which entropy is produced in a viscous fluid flow system through a porous medium. The problem under consideration is nonlinear due to the exponential nature of the rate law in Arrhenius kinetics for combustible fluids. In view of this, exact solution for the temperature field may not be possible to get. To solve the problem, we seek Adomian series solution to avoid linearization of the exponential term. The Adomian decomposition method is a straightforward way of solving all kinds of differential equations arising from many physical scenarios. It has been used extensively in the last few decades as reported in the bibliography by Rach [25], and, more recently, the method has been used in [26-31]. The plan of the article is as follows: the problem is formulated and the mathematical analysis is presented in Section 2. Section 3 of the work gives the Adomian method of solution. Graphical results are presented and interpreted in Section 4 while, in Section 5 , concluding remarks are given.

\section{Mathematical Analysis}

The steady flow of viscous incompressible reactive fluid through parallel-plate immersed in a porous medium is studied. The flow is assumed to be full-developed and driven by an applied pressure gradient. The channel wall temperatures are kept constant. Then, the balanced governing equations are [24]

$$
\begin{aligned}
& 0=-\frac{d P}{d x}+\mu \frac{d^{2} u^{\prime}}{d y^{\prime 2}}-\frac{\mu u^{\prime}}{K} \\
& 0=\frac{d^{2} T}{d y^{\prime 2}}+\frac{Q C_{0} A}{k} e^{-E / R T}+\frac{\mu}{k}\left(\frac{d u^{\prime}}{d y^{\prime}}\right)^{2}+\frac{\mu u^{\prime 2}}{k K}
\end{aligned}
$$

with the following boundary conditions:

$$
\begin{aligned}
& T(0)=T(h)=T_{0} \\
& u^{\prime}(0)=0=u^{\prime}(h) .
\end{aligned}
$$

Under these assumptions, entropy generation equation becomes

$$
E_{G}=\frac{k}{T_{0}^{2}}\left(\frac{d T}{d y^{\prime}}\right)^{2}+\frac{\mu}{T_{0}}\left(\frac{d u^{\prime}}{d y^{\prime}}\right)^{2}+\frac{\mu u^{\prime 2}}{T_{0} K} .
$$

To nondimensionalize (1)-(3), we need the following parameters and variables:

$$
\begin{aligned}
& y=\frac{y^{\prime}}{h}, \\
& u=\frac{u^{\prime}}{U M},
\end{aligned}
$$

$$
\begin{aligned}
\theta & =\frac{E\left(T-T_{0}\right)}{R T_{0}^{2}}, \\
\mathrm{Da} & =\frac{K}{h^{2}}, \\
N_{s} & =\frac{h^{2} E^{2} E_{G}}{k R^{2} T_{0}^{2}}, \\
\epsilon & =\frac{R T_{0}}{E} \\
\lambda & =\frac{Q E A C_{0} h^{2} e^{-E / R T_{0}}}{R T_{0}^{2} k}, \\
M & =-\frac{h^{2}}{\mu U} \frac{d P}{d x}, \\
\delta & =\frac{U^{2} \mu M^{2} e^{E / R T_{0}}}{\mathrm{Q} A C_{0} h^{2}}, \\
\beta^{2} & =\frac{1}{\mathrm{Da}}
\end{aligned}
$$

to get the dimensionless problems:

$$
\begin{aligned}
& \frac{d^{2} u}{d y^{2}}-\beta^{2} u=-1 ; \quad u(0)=u(1)=0 \\
& \frac{d^{2} \theta}{d y^{2}}+\lambda\left\{e^{\theta /(1+\epsilon \theta)}+\delta\left(\frac{d u}{d y}\right)^{2}+\delta \beta^{2} u^{2}\right\}=0 \\
& \quad \theta(0)=\theta(1)=0 \\
& N_{s}=\left(\frac{d \theta}{d y^{\prime}}\right)^{2}+\frac{\delta \lambda}{\epsilon}\left(\left(\frac{d u}{d y}\right)^{2}+\beta^{2} u^{2}\right) .
\end{aligned}
$$

Setting

$$
\begin{aligned}
& N_{1}=\left(\frac{d \theta}{d y}\right)^{2}, \\
& N_{2}=\frac{\delta \lambda}{\epsilon}\left(\left(\frac{d u}{d y}\right)^{2}+\beta^{2} u^{2}\right),
\end{aligned}
$$

then, the irreversibility ratio becomes

$$
\begin{aligned}
\mathrm{Be} & =\frac{N_{1}}{N_{1}+N_{2}}=\frac{1}{1+\Phi}, \\
\Phi & =\frac{N_{2}}{N_{1}} .
\end{aligned}
$$

From (9), it is evident that

$$
\mathrm{Be}= \begin{cases}0, & N_{2} \gg N_{1} \\ 0.5 & N_{1}=N_{2} \\ 1 & N_{2} \ll N_{1} .\end{cases}
$$




\section{Adomian Method of Solution}

A direct integration of (5)-(6) leads to the integral equations

$$
u(y)=\int_{0}^{y} \frac{d u(0)}{d Y} d Y+\int_{0}^{y} \int_{0}^{y}\left(\beta^{2} u\right) d Y d Y
$$

with

$$
\begin{aligned}
& \theta(y) \\
& =\int_{0}^{y} \frac{d \theta(0)}{d Y} d Y \\
& \quad-\int_{0}^{y} \int_{0}^{y} \lambda\left\{e^{\theta /(1+\epsilon \theta)}+\delta\left(\frac{d u}{d Y}\right)^{2}+\delta \beta^{2} u^{2}\right\} d Y d Y .
\end{aligned}
$$

Due to the exponential nonlinearity in (12), we now define a series of functions defined by

$$
\begin{aligned}
& u(y)=\sum_{n=0}^{\infty} u_{n}(y), \\
& \theta(y)=\sum_{n=0}^{\infty} \theta_{n}(y) .
\end{aligned}
$$

Substituting (13) into the integral equations (11)-(12), we obtain

$$
\begin{aligned}
& \sum_{n=0}^{\infty} u_{n}(y)=\int_{0}^{y} \frac{d u(0)}{d Y} d Y \\
& +\int_{0}^{y} \int_{0}^{y}\left(\beta^{2} \sum_{n=0}^{\infty} \theta_{n}(y)\right) d Y d Y, \\
& \sum_{n=0}^{\infty} \theta_{n}(y)=\int_{0}^{y} \frac{d \theta(0)}{d Y} d Y \\
& -\int_{0}^{y} \int_{0}^{y} \lambda\left\{\operatorname{Exp}\left(\frac{\sum_{n=0}^{\infty} \theta_{n}(y)}{1+\epsilon \sum_{n=0}^{\infty} \theta_{n}(y)}\right)\right. \\
& +\delta\left(\frac{d}{d Y}\left(\sum_{n=0}^{\infty} u_{n}(y)\right)\right)^{2} \\
& \left.+\delta \beta^{2}\left(\sum_{n=0}^{\infty} u_{n}(y)\right)^{2}\right\} d Y d Y .
\end{aligned}
$$

The nonlinear term in (15) represented by

$$
B_{n}=\operatorname{Exp}\left(\frac{\sum_{n=0}^{\infty} \theta_{n}(y)}{1+\epsilon \sum_{n=0}^{\infty} \theta_{n}(y)}\right)
$$

is expanded by Taylor's series to get the following Adomian polynomials:

$$
\begin{aligned}
& B_{0}=e^{\theta_{0} /\left(1+\epsilon \theta_{0}\right)} \\
& B_{1}=\frac{\theta_{1}}{\left(1+\epsilon \theta_{1}\right)^{2}} e^{\theta_{0} /\left(1+\epsilon \theta_{0}\right)}
\end{aligned}
$$

$$
\begin{aligned}
B_{2} & =\frac{\left\{\left(1-2 \epsilon-2 \epsilon^{2} \theta_{0}\right) \theta_{1}^{2}+2\left(1+\epsilon\left(\theta_{0}\right)^{2} \theta_{2}\right)\right\}}{2\left(1+\epsilon \theta_{1}\right)^{4}} \\
& \cdot e^{\theta_{0} /\left(1+\epsilon \theta_{0}\right)}
\end{aligned}
$$

The zeroth-order components of the series solutions (14) and (15) are

$$
\begin{aligned}
& u_{0}(y)=a_{0} y-\frac{y^{2}}{2} \\
& \theta_{0}(y)=0 .
\end{aligned}
$$

Since the integral of a continuous function is continuous, then each term of the series can be uniquely determined by

$$
\begin{aligned}
& u_{n+1}(y)=\beta^{2} \int_{0}^{y} \int_{0}^{y} u_{n} d Y d Y \quad n \geq 1 \\
& \theta_{1}(y) \\
& \quad=\int_{0}^{y} a_{1} d Y \\
& -\lambda \int_{0}^{y} \int_{0}^{y}\left(B_{0}+\delta\left(\frac{d u}{d Y}\right)^{2}+\delta \beta^{2} u^{2}\right) d Y d Y \\
& \theta_{n+1}(y)=-\lambda \int_{0}^{y} \int_{0}^{y} B_{n} d Y d Y \quad n \geq 1,
\end{aligned}
$$

where $d u(0) / d Y=a_{0}$ and $d \theta(0) / d Y=a_{1}$ are the parameters to be determined.

Then, (17)-(19) are evaluated using MATHEMATICA and the solutions are obtained as finite series:

$$
\begin{aligned}
& u(y)=\sum_{n=0}^{m} u_{n}(y), \\
& \theta(y)=\sum_{n=0}^{m} \theta_{n}(y) .
\end{aligned}
$$

The series solutions are shown to be convergent and twice differentiable (see Tables 1 and 2). Next, we establish the 
uniqueness solution of (20). It is well known that the Lipschitz condition is sufficient for the uniqueness of solution. Therefore, we first seek for a Lipschitz constant $\phi$ such that

$$
\|F(\theta)-F(\bar{\theta})\| \leq \phi\|\theta(y)-\theta(\bar{y})\|
$$

is satisfied. To do this, the boundary-valued problems (6) are converted to system first-order differential equations by introducing the following transformations:

$$
\begin{aligned}
& y_{1}=y, \\
& y_{2}=u(y), \\
& y_{3}=\theta(y), \\
& y_{4}=u^{\prime}(y), \\
& y_{5}=\theta^{\prime}(y) .
\end{aligned}
$$

$$
\frac{d f_{i}}{d y_{j}}=\left(\begin{array}{ccccc}
0, & 0, & 0, & 0, & 0 \\
0, & 0, & 0, & 1, & 0 \\
0, & 0, & 0, & 0, & 1 \\
0, & 0, & \beta^{2} & 1, & 1 \\
0, & -2 \lambda \delta \beta^{2} y_{2} & -\frac{\lambda}{1+\epsilon y_{4}}\left(1-\frac{\epsilon y_{3}}{1+\epsilon y_{3}}\right) e^{y_{3} /\left(1+\varepsilon y_{3}\right)} & -2 \delta \lambda y_{3} & 0
\end{array}\right)
$$

With (22), (6) can now be written as since $d f_{i} / d y_{j}, i, j=1,2, \ldots, 5$, exist and are continuous in the domain $[0,1]$. Hence, the Lipschitz constant $\phi$ with the property

$$
\left|\frac{d f_{i}}{d y_{i}}\right| \leq \phi
$$

exists.

Uniqueness Analysis. The Adomian series solutions (20) of the nonlinear problem (6) converges if $0<\alpha \ll 1$ and $\left|\theta_{0}(y)\right|<$ $\infty$, where $\alpha=\lambda K Y^{2} / 2$.

Proof. Let $(C[J],\|\cdot\|)$ be a Banach space for all continuous functions on $J$ with the norm

$$
\|F(Y)\|=\max _{\forall y \in J}|F(Y)|
$$

$$
\begin{aligned}
& \left(\begin{array}{l}
y_{1} \\
y_{2} \\
y_{3} \\
y_{4} \\
y_{5}
\end{array}\right)^{\prime}=\left(\begin{array}{c}
1 \\
y_{4} \\
y_{5} \\
\beta^{2} y_{2}-1 \\
-\lambda\left(e^{y_{3} /\left(1+\epsilon y_{3}\right)}+\delta\left(y_{4}^{2}+\beta^{2} y_{2}^{2}\right)\right)
\end{array}\right) \\
& =\left(\begin{array}{c}
f_{1}\left(y_{1}, y_{2}, \ldots, y_{5}\right) \\
f_{2}\left(y_{1}, y_{2}, \ldots, y_{5}\right) \\
f_{3}\left(y_{1}, y_{2}, \ldots, y_{5}\right) \\
f_{4}\left(y_{1}, y_{2}, \ldots, y_{5}\right) \\
f_{5}\left(y_{1}, y_{2}, \ldots, y_{5}\right)
\end{array}\right) ; \\
& \left(\begin{array}{l}
y_{1}(0) \\
y_{2}(0) \\
y_{3}(0) \\
y_{4}(0) \\
y_{5}(0)
\end{array}\right)=\left(\begin{array}{c}
0 \\
0 \\
0 \\
c_{1} \\
c_{2}
\end{array}\right),
\end{aligned}
$$

where $c_{i}(i=1,2)$, the guess values that will ensure the boundary conditions, are satisfied. Then,

Let $\theta(y)$ and $\theta(\bar{y})$ be any two solutions of the integral equation (12); then,

$$
|\theta(y)-\theta(\bar{y})|=\left|-\int_{0}^{y} \int_{0}^{y} \lambda\{F(\theta)-F(\bar{\theta})\} d Y d Y\right| .
$$

This implies that

$$
|\theta(y)-\theta(\bar{y})| \leq \int_{0}^{y} \int_{0}^{y}|\lambda\{F(\theta)-F(\bar{\theta})\}| d Y d Y .
$$

In view of (21), we then have

$$
\begin{aligned}
|\theta(y)-\theta(\bar{y})| & \leq \int_{0}^{y} \int_{0}^{y}|\theta(y)-\theta(\bar{y})| d Y d Y \\
& \leq|\theta(y)-\theta(\bar{y})| \int_{0}^{y} \int_{0}^{y} \lambda \phi d Y d Y \\
& \leq \frac{\lambda \phi Y^{2}}{2}|\theta(y)-\theta(\bar{y})| .
\end{aligned}
$$


TABLE 1: Uniqueness results $m=3, \beta=1$.

\begin{tabular}{lccc}
\hline$y$ & $u$-exact & $u$-Adomian & Error \\
\hline 0 & 0 & 0 & 0 \\
0.1 & 0.0412846 & 0.0412847 & $8.58689 \times 10^{-8}$ \\
0.2 & 0.072741 & 0.072742 & $1.72597 \times 10^{-7}$ \\
0.3 & 0.0953855 & 0.0953858 & $2.61030 \times 10^{-7}$ \\
0.4 & 0.108743 & 0.108744 & $3.51816 \times 10^{-7}$ \\
0.5 & 0.113181 & 0.113182 & $4.44491 \times 10^{-7}$ \\
0.6 & 0.108743 & 0.108744 & $5.34572 \times 10^{-7}$ \\
0.7 & 0.0953855 & 0.0953861 & $6.06498 \times 10^{-7}$ \\
0.8 & 0.0729741 & 0.0729747 & $6.1915 \times 10^{-7}$ \\
0.9 & 0.0412846 & 0.0412851 & $4.79648 \times 10^{-7}$ \\
1 & 0 & 0 & 0 \\
\hline
\end{tabular}

TABLE 2: Convergence results $\beta=1=\lambda=\delta, \epsilon=0.1$.

\begin{tabular}{lc}
\hline$N$ & $b 1$ \\
\hline 0 & 0 \\
1 & 0.537883 \\
2 & 0.590362 \\
3 & 0.589506 \\
4 & 0.589078 \\
5 & 0.589096 \\
6 & 0.589100 \\
7 & 0.589100 \\
8 & 0.589100 \\
\hline
\end{tabular}

Let $\alpha=\lambda \phi Y^{2} / 2$; then,

$$
|\theta(y)-\theta(\bar{y})| \leq \alpha|\theta(y)-\theta(\bar{y})|
$$

or

$$
(1-\alpha)|\theta(y)-\theta(\bar{y})| \leq 0 .
$$

Hence, the problem will have a unique solution whenever $0<$ $\alpha \ll 1$ and as such

$$
\theta(y)=\theta(\bar{y})
$$

Kindly see Tables 1 and 2 for the numerical results.

\section{Results and Discussion}

In this section, the effects of pertinent fluid parameters on the velocity and temperature profiles are shown graphically. Figure 1 shows the effect of medium porosity on the entropy generation rate. Since an increase in the porous permeability parameter implies a decrease in the medium porosity, this caused a decreased flow and heat trapping strategy. The net effect is seen here; that is, entropy production increases only in the centerline of the channel while it decreases at the walls. Figure 2 represents the effect of activation energy parameter on the entropy generation rate. Since activation energy

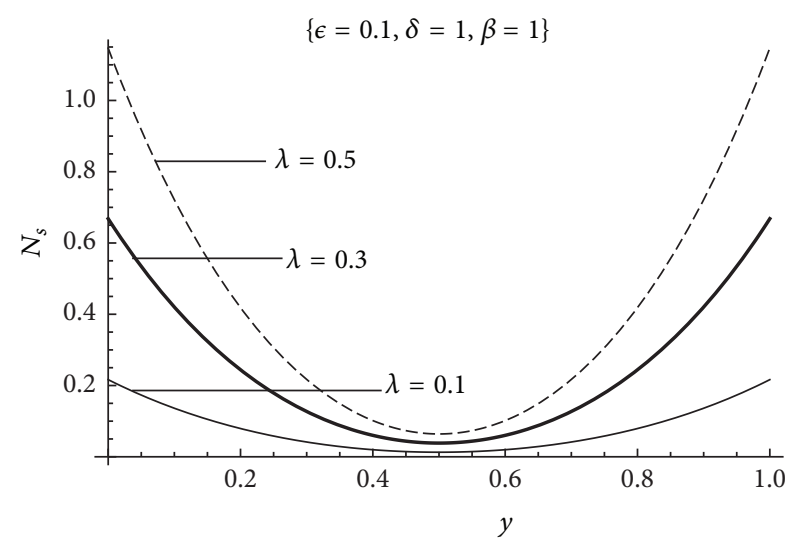

FIGURE 1: Entropy generation rate for different porosity permeability parameter.

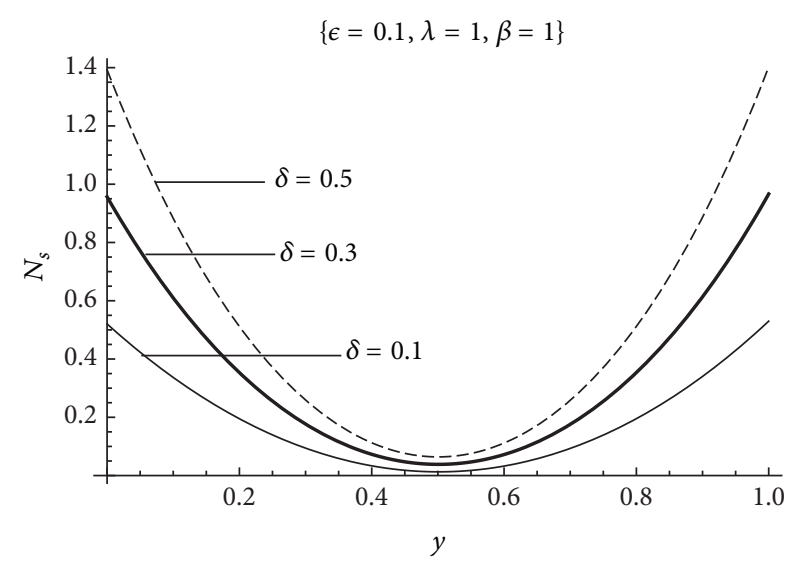

FIGURE 2: Entropy generation rate for different activation energy parameter.

decreases the fluid temperature, it is therefore expected to decrease the entropy generated in the flow region. This is true since an increase in the Frank-Kamenetskii parameter is known to enhance the fluid temperature. Therefore, by increasing values of this parameter, entropy generated is expected to be on the increase as shown in Figure 3. In Figure 4, the effect of the viscous heating parameter is shown. The result shows that entropy generation rate increases with increasing values of the viscous heating parameter due to frictional interaction in the fluid layers. Figure 5 represents the Bejan number's variations with the activation energy. From the plot, as the activation energy parameter increases, heat irreversibility due to heat transfer decreases. As a result, fluid friction irreversibility dominates over heat transfer irreversibility within the porous medium. Moreover, as the porous permeability parameter increases in Figure 6, there are reduced flow and fluid temperature rises. The net effect shows that heat transfer irreversibility dominates over irreversibility due to fluid friction. Similar behaviour is observed in Figure 7 as the exothermic Frank-Kamenetskii parameter increases. Finally, as viscous heating parameter increases, the viscous heat dissipation increases in the flow 


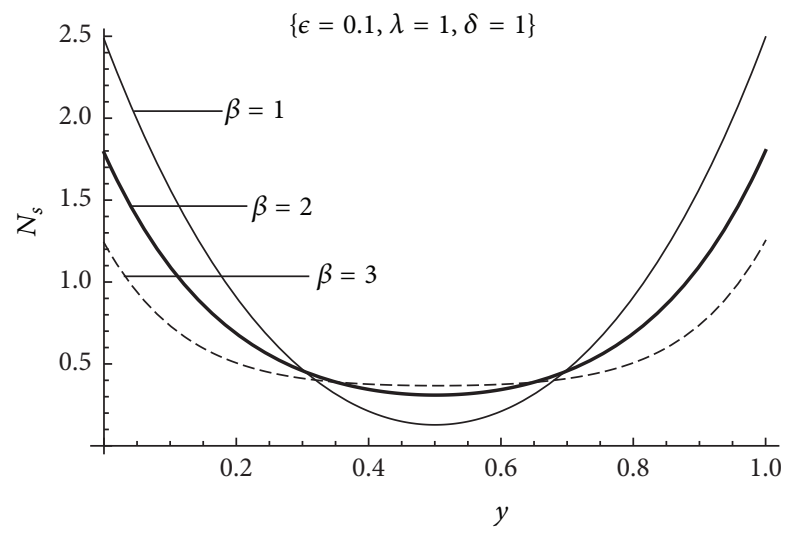

Figure 3: Entropy generation rate for different Frank-Kamenetskii parameter.

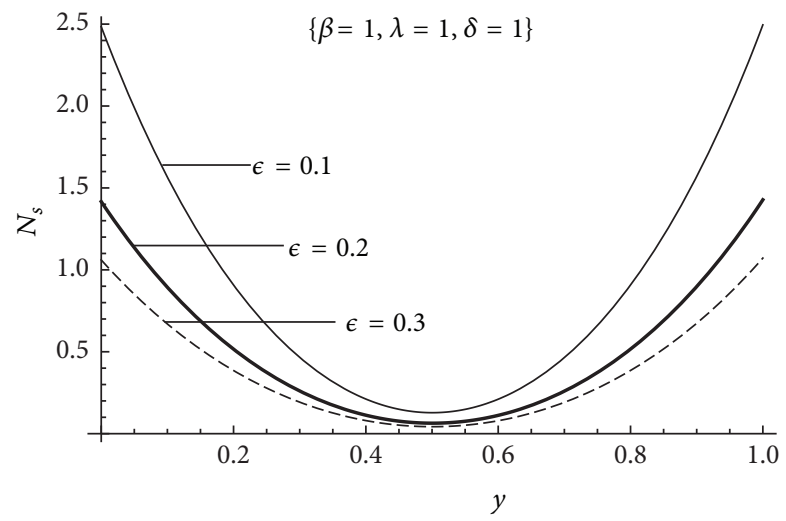

Figure 4: Entropy generation rate for different viscous heating parameter.

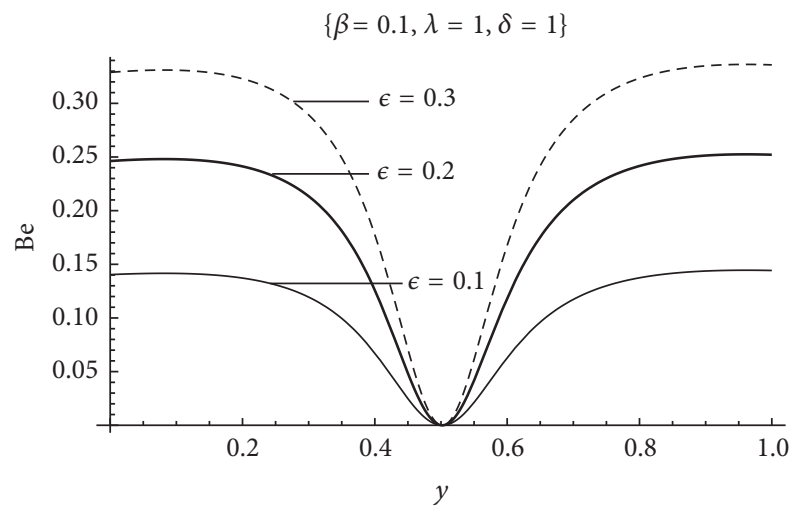

FIGURE 5: Bejan number at different activation energy parameter.

channel, and fluid friction irreversibility dominates over heat transfer irreversibility as observed in Figure 8.

\section{Conclusion}

The entropy generation rate in the flow of reactive fluid through a porous medium has been investigated. Adomian

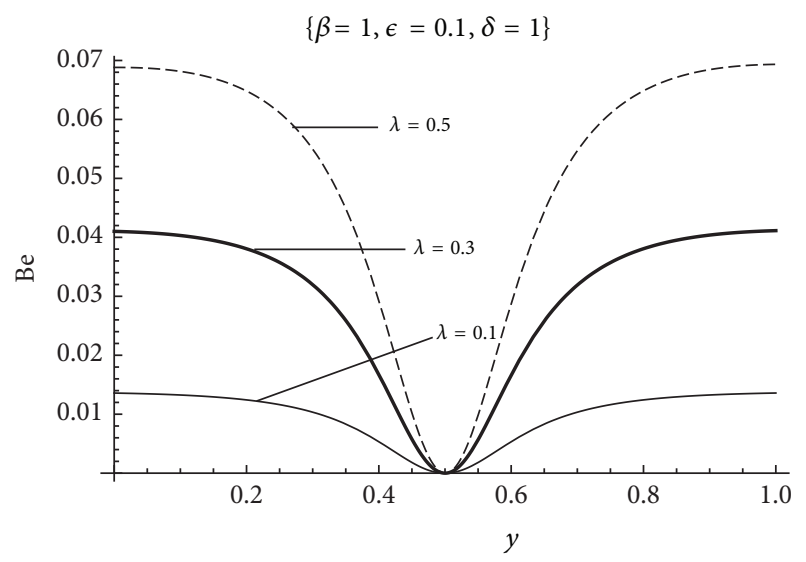

FIGURE 6: Bejan number at different porosity permeability parameter.

$$
\{\beta=1, \lambda=1, \epsilon=0.1\}
$$

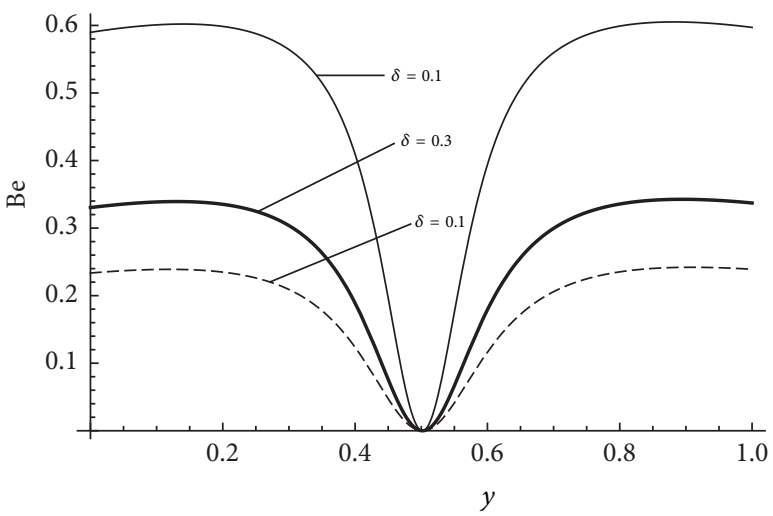

FIGURE 7: Bejan number at different Frank-Kamenetskii parameter.

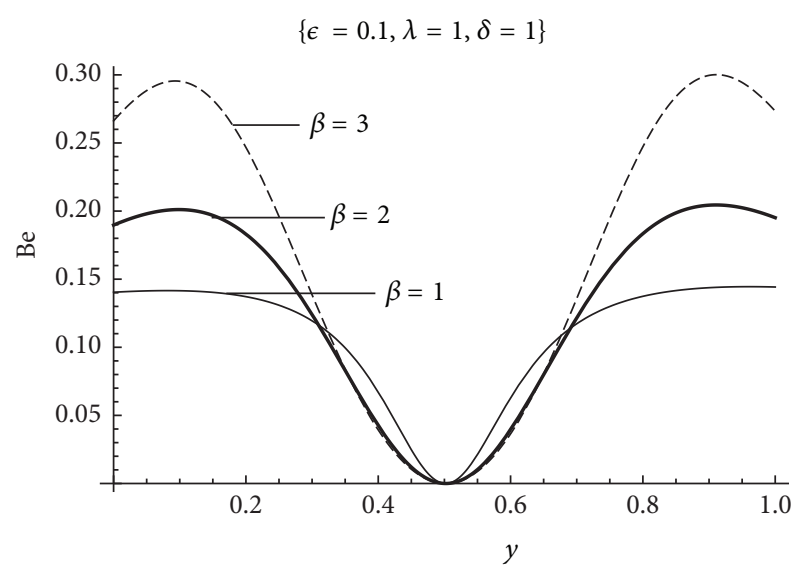

FIGURE 8: Bejan number at different viscous heating parameter.

decomposition method is used to obtain approximate solution to the strongly nonlinear boundary-valued problem for the dimensionless energy equation. The main contributions to knowledge from the present analysis are as follows: a reactive fluid flowing through a porous medium, entropy generation is minimum at the centerline of the channel; 
as a result, fluid friction irreversibility dominates over heat transfer irreversibility in the centerline. Secondly, porous medium with low permeability is one major factor that depletes the useful available energy in a thermofluid.

\section{Nomenclature}

$\begin{array}{ll}T: & \text { Fluid temperature } \\ P: & \text { Pressure } \\ T_{0}: & \text { Wall temperature } \\ k: & \text { Thermal conductivity of the material } \\ K: & \text { Porous permeability respectively } \\ \mu: & \text { Dynamic viscosity } \\ Q: & \text { Heat of reaction } \\ A: & \text { Rate constant } \\ E: & \text { Activation energy } \\ R: & \text { Universal gas constant } \\ C_{0}: & \text { Initial concentration of the reactant } \\ & \text { species } \\ h: & \text { Channel half width } \\ (x, y): & \text { Cartesian coordinates system } \\ U: & \text { Fluid characteristic velocity } \\ M: & \text { Dimensionless axial pressure gradient } \\ \lambda: & \text { Frank-Kamenetskii parameter } \\ \epsilon: & \text { Activation energy parameter } \\ \delta: & \text { Viscous heating parameter } \\ \beta: & \text { Porous medium permeability parameter } \\ \text { Da: } & \text { Darcy number, respectively } \\ E_{G}, N_{s}: & \text { Dimensional and dimensionless entropy } \\ & \text { generation. }\end{array}$

\section{Competing Interests}

The authors declare that there is no conflict of interests regarding the publication of this paper.

\section{References}

[1] A. Bejan, "Second law analysis in heat transfer," Energy, vol. 5, no. 8-9, pp. 720-732, 1980.

[2] I. T. Al-Zaharnah and B. S. Yilbas, "Thermal analysis in pipe flow: influence of variable viscosity on entropy generation," Entropy, vol. 6, no. 3, pp. 344-363, 2004.

[3] O. M. Haddad, M. K. Alkam, and M. T. Khasawneh, "Entropy generation due to laminar forced convection in the entrance region of a concentric annulus," Energy, vol. 29, no. 1, pp. 35$55,2004$.

[4] A. Kahraman and M. Yürüsoy, "Entropy generation due to nonnewtonian fluid flow in annular pipe with relative rotation: constant viscosity case," Journal of Theoretical and Applied Mechanics, vol. 46, no. 1, pp. 69-83, 2008.

[5] Y. Aksoy, "Effects of couple stresses on the heat transfer and entropy generation rates for a flow between parallel plates with constant heat flux," International Journal of Thermal Sciences, vol. 107, pp. 1-12, 2016.

[6] T. W. Ting, Y. M. Hung, and N. Guo, "Entropy generation of viscous dissipative nanofluid flow in thermal non-equilibrium porous media embedded in microchannels," International Journal of Heat and Mass Transfer, vol. 81, pp. 862-877, 2015.
[7] W. A. Khan and R. S. R. Gorla, "Second law analysis for free convection in non-Newtonian fluids over a horizontal plate embedded in a porous medium: (prescribed heat flux)," Brazilian Journal of Chemical Engineering, vol. 29, no. 3, pp. 511518, 2012.

[8] R. Revellin, S. Lips, S. Khandekar, and J. Bonjour, "Local entropy generation for saturated two-phase flow," Energy, vol. 34, no. 9, pp. 1113-1121, 2009.

[9] F. Hedayati, A. Malvandi, and D. D. Ganji, "Second-law analysis of fluid flow over an isothermal moving wedge," Alexandria Engineering Journal, vol. 53, no. 1, pp. 1-9, 2014.

[10] A. S. Butt and A. Ali, "Effects of magnetic field on entropy generation in flow and heat transfer due to a radially stretching surface," Chinese Physics Letters, vol. 30, no. 2, Article ID 024701, 2013.

[11] S. O. Adesanya and O. D. Makinde, "Thermodynamic analysis for a third grade fluid through a vertical channel with internal heat generation," Journal of Hydrodynamics, vol. 27, no. 2, pp. 264-272, 2015.

[12] S. O. Adesanya and O. D. Makinde, "Entropy generation in couple stress fluid flow through porous channel with fluid slippage," International Journal of Exergy, vol. 15, no. 3, pp. 344362, 2014.

[13] S. O. Adesanya and O. D. Makinde, "Effects of couple stresses on entropy generation rate in a porous channel with convective heating," Computational \& Applied Mathematics, vol. 34, no. 1, pp. 293-307, 2015.

[14] S. Mahmud and R. A. Fraser, "Thermodynamic analysis of flow and heat transfer inside channel with two parallel plates," Exergy, vol. 2, no. 3, pp. 140-146, 2002.

[15] S. H. Tasnim, M. Shohel, and M. A. H. Mamun, "Entropy generation in a porous channel with hydromagnetic effect," Exergy, vol. 2, no. 4, pp. 300-308, 2002.

[16] I. Ozkol, G. Komurgoz, and A. Arikoglu, "Entropy generation in laminar natural convection from a constant temperature vertical plate in an infinite fluid," Proceedings of the Institution of Mechanical Engineers, Part A: Journal of Power and Energy, vol. 221, no. 5, pp. 609-616, 2007.

[17] S. O. Adesanya and O. D. Makinde, "Irreversibility analysis in a couple stress film flow along an inclined heated plate with adiabatic free surface," Physica A, vol. 432, pp. 222-229, 2015.

[18] J. Srinivas, J. R. Murthy, and A. J. Chamkha, "Analysis of entropy generation in an inclined channel flow containing two immiscible micropolar fluids using HAM," International Journal of Numerical Methods for Heat \& Fluid Flow, vol. 26, no. 3-4, pp. 1027-1049, 2016.

[19] J. Srinivas and J. V. Ramana Murthy, "Second law analysis of the flow of two immiscible micropolar fluids between two porous beds," Journal of Engineering Thermophysics, vol. 25, no. 1, pp. 126-142, 2016.

[20] O. D. Makinde, T. Chinyoka, and L. Rundora, "Unsteady flow of a reactive variable viscosity non-Newtonian fluid through a porous saturated medium with asymmetric convective boundary conditions," Computers \& Mathematics with Applications, vol. 62, no. 9, pp. 3343-3352, 2011.

[21] L. Rundora and O. D. Makinde, "Effects of suction/injection on unsteady reactive variable viscosity non-Newtonian fluid flow in a channel filled with porous medium and convective boundary conditions," Journal of Petroleum Science and Engineering, vol. 108, pp. 328-335, 2013.

[22] L. Rundora and O. D. Makinde, "Effects of Navier slip on unsteady flow of a reactive variable viscosity non-Newtonian 
fluid through a porous saturated medium with asymmetric convective boundary conditions," Journal of Hydrodynamics, vol. 27, no. 6, pp. 934-944, 2015.

[23] O. A. Bég, H. S. Takhar, R. Bhargava, S. Rawat, and V. R. Prasad, "Numerical study of heat transfer of a third grade viscoelastic fluid in non-Darcy porous media with thermophysical effects," Physica Scripta, vol. 77, no. 6, Article ID 065402, 2008.

[24] O. D. Makinde, "Thermal ignition in a reactive viscous flow through a channel filled with a porous medium," Journal of Heat Transfer, vol. 128, no. 6, pp. 601-604, 2006.

[25] R. Rach, "A bibliography of the theory and applications of the Adomian decomposition method, 1961-2011," Kybernetes, vol. 41, no. 7-8, pp. 1087-1153, 2012.

[26] G. Adomian and R. Rach, "Modified decomposition solution of nonlinear partial differential equations," Applied Mathematics Letters, vol. 5, no. 6, pp. 29-30, 1992.

[27] G. Adomian and R. Rach, "Analytic solution of nonlinear boundary value problems in several dimensions by decomposition," Journal of Mathematical Analysis and Applications, vol. 174, no. 1, pp. 118-137, 1993.

[28] M. Sheikholeslami, D. D. Ganji, H. R. Ashorynejad, and H. B. Rokni, "Analytical investigation of Jeffery-Hamel flow with high magnetic field and nanoparticle by Adomian decomposition method," Applied Mathematics and Mechanics, vol. 33, no. 1, pp. 25-36, 2012.

[29] M. R. Mohyuddin, "Resonance and viscoelastic poiseuille flow in a porous medium," Journal of Porous Media, vol. 9, no. 8, pp. 799-806, 2006.

[30] M. R. Mohyuddin, "MHD flow and heat transfer for second grade fluid in a porous medium with modified darcy's," International Journal of Fluid Mechanics Research, vol. 34, no. 5, pp. 462-474, 2007.

[31] S. Asghar, M. R. Mohyuddin, and T. Hayat, "Effects of Hall current and heat transfer on flow due to a pull of eccentric rotating disks," International Journal of Heat and Mass Transfer, vol. 48, no. 3-4, pp. 599-607, 2005. 


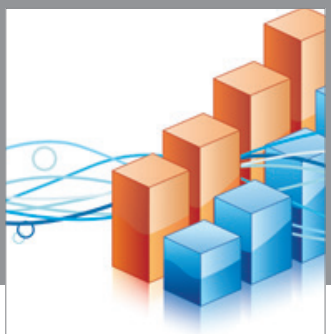

Advances in

Operations Research

vatem alat4

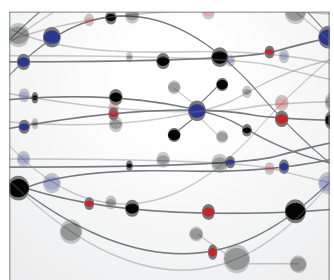

\section{The Scientific} World Journal
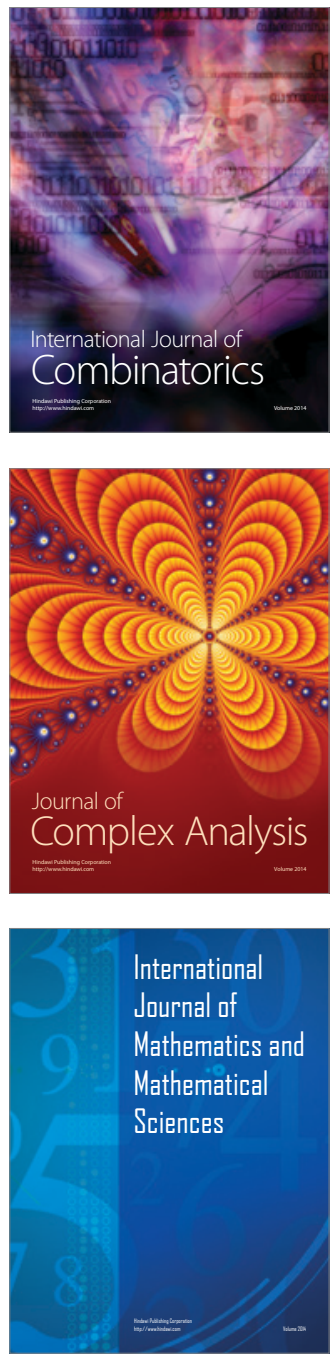
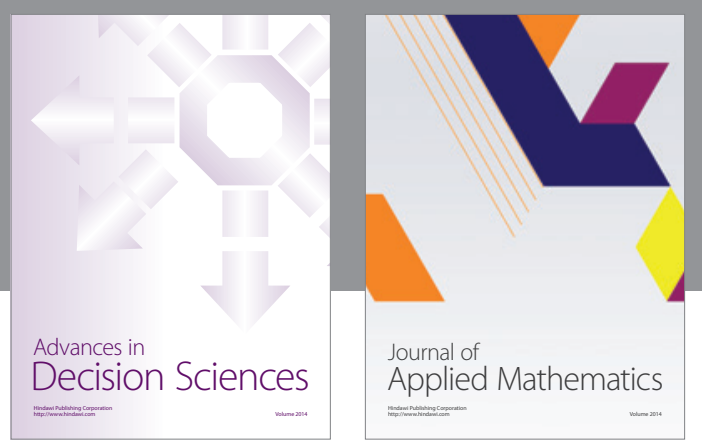

Algebra

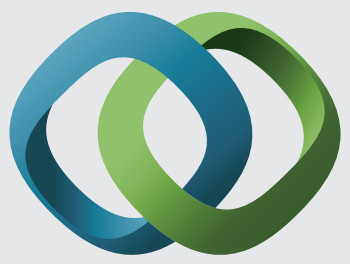

\section{Hindawi}

Submit your manuscripts at

http://www.hindawi.com
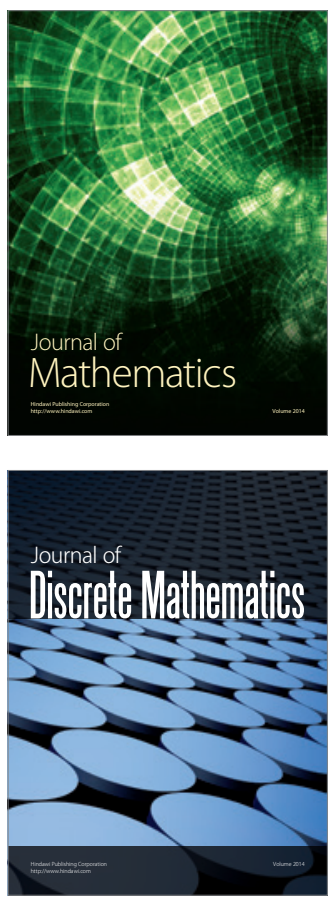

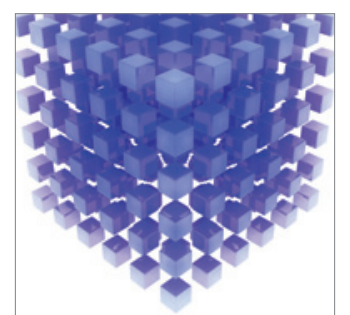

Mathematical Problems in Engineering
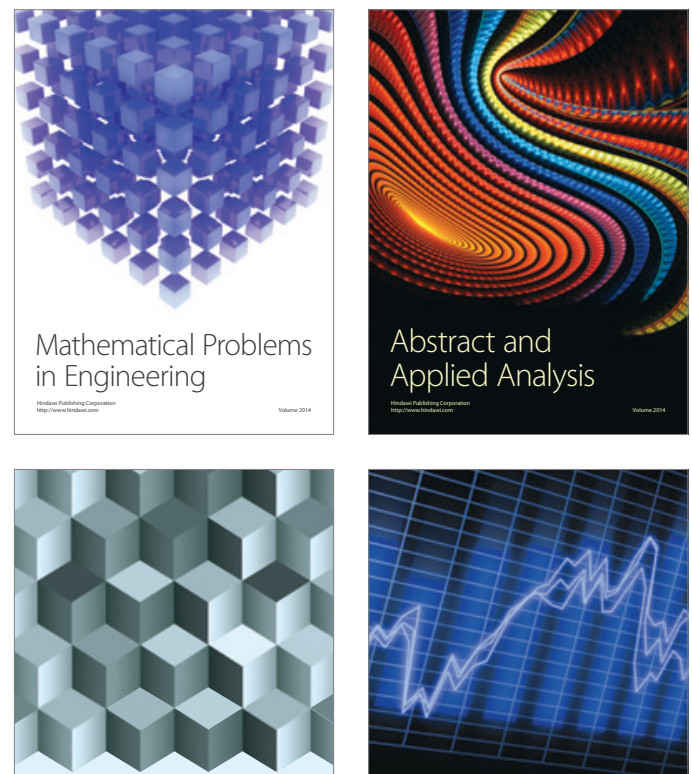

Journal of

Function Spaces

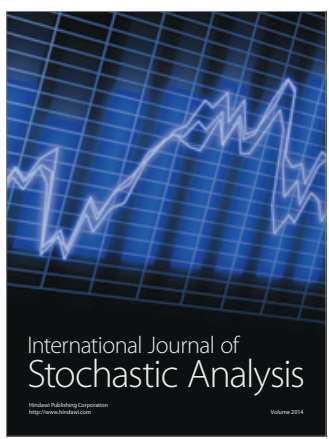

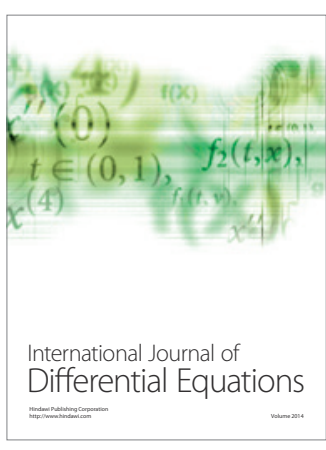
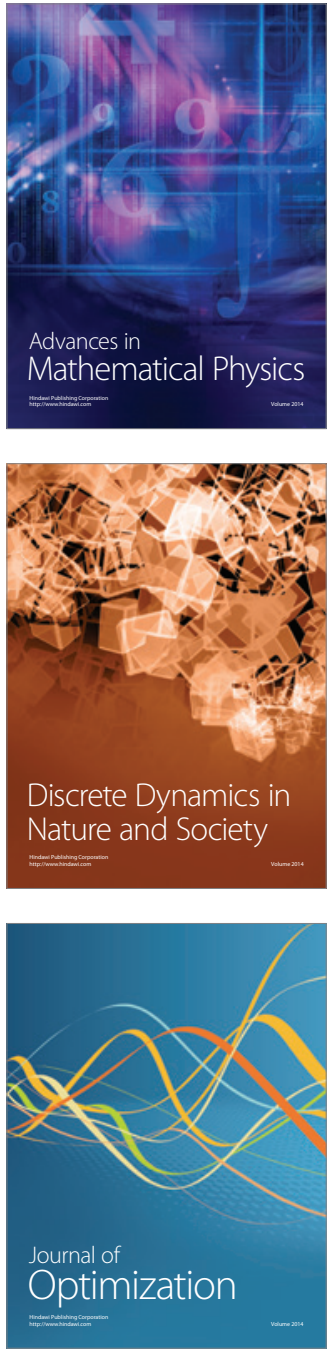